\title{
Spatial-frequency-contingent color aftereffects: Adaptation with two-dimensional stimulus patterns
}

\author{
WILLIAM R. WEBSTER, R. H. DAY, and OWEN GILLIES \\ Monash University, Clayton, Victoria, Australia \\ and \\ BORIS CRASSINI \\ Deakin University, Victoria, Australia
}

\begin{abstract}
The spatial-frequency theory of vision has been supported by adaptation studies using checkerboards in which contingent color aftereffects (CAEs) were produced at fundamental frequencies oriented at $45^{\circ}$ to the edges. A replication of this study failed to produce CAEs at the orientation of either the edges or the fundamentals. Using a computer-generated display, no CAEs were produced by adaptation of a square or an oblique checkerboard. But when one type of checkerboard (4 cpd) was adapted alone, CAEs were produced on the adapted checkerboard and on sine-wave gratings aligned with the fundamental and third harmonics of the checkerboard spectrum. Adaptation of a coarser checkerboard $(0.80 \mathrm{cpd})$ produced CAEs aligned with both the edges and the harmonic frequencies. With checkerboards of both frequencies, CAEs were also found on the other type of checkerboard that had not been adapted. This observation raises problems for any edgedetector theory of vision, because there was no adaptation to edges. It was concluded that spatialfrequency mechanisms are operating at both low- and high-spatial frequencies and that an edge mechanism is operative at lower frequencies. The implications of these results are assessed for other theories of spatial vision.
\end{abstract}

R. L. De Valois and K. K. De Valois (1980) have pointed out that there are two dominant theories of early visual processing: feature detection (or edge and bar) theory and linear spatial-frequency theory. Kelly and Magnuski (1975) and Kelly $(1976,1979)$ extended spatial-frequency analysis to stimuli with two-dimensional luminance profiles, such as checkerboards. By analysis, Kelly (1976) showed that there is no Fourier energy parallel to the edges of checkerboards; rather, the energy is located at the orientations of the fundamental and harmonic frequencies of the checkerboard spectrum. May and Matteson (1976) and Green, Corwin, and Zemon (1976) predicted from this theory that a McCollough effect (McCollough, 1965) would occur on gratings only at the orientation of the fundamentals (which are located at $45^{\circ}$ from the edges) after adaptation with colored checkerboards. They confirmed this prediction, and R. L. De Valois and K. K. De Valois (1980) have pointed out the importance of these results for spatial-frequency theory.

Using the color-cancellation technique of White (1976), May and his collaborators (May, Agamy, \& Matteson, 1978; May, Matteson, Agamy, \& Castellanos, 1978) con-

The authors wish to thank Keith Willenberg and John Cassell for computer programming. We also wish to thank V. Kohout for photography, Rosemary Williams for illustrations, and Moyra Farrington for typing. This research was supported by a grant from the Australian Research Committee. Correspondence should be addressed to William R. Webster, Department of Psychology, Monash University, Clayton, Victoria 3168, Australia. firmed these observations with high-frequency checkerboards and gratings (3-7 cycles per degree [cpd]) but failed to do so in experiments with low-frequency checkerboards and gratings $(0.40,0.80$, and $1.50 \mathrm{cpd})$. Importantly, with 0.80 -cpd checkerboards, they found McCollough effects on gratings aligned with the edges of the checkerboard and suggested that these results implied an edge mechanism operating at some lower frequencies.

The general trend of these psychophysical results has been supported by single-unit studies of visual cortex. K. K. De Valois, R. L. De Valois, and Yund (1979) found that a cell would not respond when its receptive field (RF) was aligned with the edges of a checkerboard but would respond when the RF was aligned in the orientations of the fundamental and the third harmonic. Smith (1977) and Tyler (1977) have criticized the conclusion of May and Matteson (1976) by suggesting that the diagonals of the checks in a checkerboard at $45^{\circ}$ to the edges fall on a common line. This alignment produces stimuli that could excite an edge detector. MacKay (1981) has put forward a similar argument against the neurophysiological studies (K. K. De Valois et al., 1979). However, K. K. De Valois et al. (1979) also showed the effect at the orientations of the third harmonic, which would not support the common-diagonal explanation.

The general aim of these experiments was to reexamine the McCollough effect with checkerboards, with particular emphasis on the higher harmonics. It was felt that the broad spatial-frequency tuning for the McCollough 
effect shown in our companion paper (Day, Webster, Gillies, \& Crassini, 1992), taken in conjunction with the broad orientation tuning for checkerboards (Green, 1980), makes it difficult to understand how spatial-frequency mechanisms could generate selective aftereffects after adaptation with both square and oblique checkerboards. The first experiment was concerned with establishing the robustness of the checkerboard effect.

\section{EXPERIMENT 1}

The purpose of Experiment 1 was to replicate the checkerboard results described above using experimental conditions similar to those of May and Matteson (1976) and Green et al. (1976). Observers were adapted with slide-presented checkerboards with a check size of either 2.0 or $5.0 \mathrm{cpd}$. The occurrence of color aftereffects (CAEs) was tested with test slides and also with a computer graphics system, which allowed the presentation of sine-wave gratings as well as square-wave gratings. Since neither May and Matteson (1976) nor Green et al. (1976) specified the contrast of their stimuli, except to say that it was "high contrast," the maximum level of contrast was employed in the present experiment to give conditions comparable with their previous work.

\section{Method}

Subjects. Eight students with normal visual acuity and color vision, as assessed by the Ishihara (1964) test, participated as subjects.

Apparatus and Stimulus Patterns. Adaptation and test patterns were presented as images on a white screen using a Kodak Carousel projector. The subjects were also presented with test patterns on a computer-controlled color-graphics system based on a DEC-LSI-73 computer. This system and its method of operation has already been described in detail in a companion paper (Day et al., 1992). Adaptation patterns were prepared as slides by mounting negatives of square checkerboard (SCB) and diagonal checkerboard (DCB) patterns together with Wratten filters 26 (red) and 41 (green). Red patterns were presented at an average luminance of $19.8 \mathrm{~cd} / \mathrm{m}^{2}$ and 92.5\% contrast; green stimuli were presented at an average luminance of $33.3 \mathrm{~cd} / \mathrm{m}^{2}$ and $92.5 \%$ contrast. The checkerboards were either 2 or $5 \mathrm{cpd}$ in spatial frequency at viewing distances of 1.0 or $2.5 \mathrm{~m}$, respectively. The single test stimulus was a slide that consisted of a split-field pattern made up of an SCB on the left half and a DCB on the right (average luminance $39.7 \mathrm{~cd} / \mathrm{m}^{2}, 92.5 \%$ contrast); both halves were either 2 or $5 \mathrm{cpd}$ in spatial frequency. The slide-presented adaptation and test patterns subtended $20^{\circ}$ vertically $\times 25^{\circ}$ horizontally. Ten test patterns were presented on the colorgraphics system. Four were split-field grating patterns consisting of vertical gratings on the left and horizontal ones on the right. Two gratings were square waves, and two were sine waves. One grating of each type was $2.0 \mathrm{cpd}$ and the other was $2.8 \mathrm{cpd}$ when viewed at $1 \mathrm{~m}$. The other four gratings were also split fields, but with the left containing $45^{\circ}$ obliques and the right $135^{\circ}$ obliques. The same parameters as the first four gratings were spread across these gratings. When all eight gratings were presented at $2.5 \mathrm{~m}$, their spatial frequencies were 5.0 and $7.1 \mathrm{cpd}$. The gratings tested at 2.8 and $7.1 \mathrm{cpd}$ were the fundamental frequencies of the two sizes of checkerboards at $\sqrt{2} \times$ checkerboard frequency (Kelly, 1976). The remaining two achromatic test stimuli were full-field checkerboards (SCB, DCB), with check sizes of 2.0 and $5.0 \mathrm{cpd}$ at the 1.0 and $2.5 \mathrm{~m}$ viewing distances, respectively. All test patterns were $16.7 \mathrm{~cd} / \mathrm{m}^{2}$ in luminance and $97 \%$ in contrast.
Procedure. All observers were tested in two sessions, one week apart. In the first session, they made judgments seated at $1.0 \mathrm{~m}$ from the patterns and in the second at $2.5 \mathrm{~m}$. In each session, the observers made quantitative nulling judgments of the computerpresented stimuli and then qualitative judgments of the slide test patterns. They were then adapted for a total period of $10 \mathrm{~min}$ to the colored patterns in which the checkerboards were presented in alternation for $10 \mathrm{sec}$. The colors of the checkerboards were counterbalanced: half of the subjects were adapted with red squares and green diamonds, and half were adapted with the reverse. After adaptation, there was a rest period of 2 min to allow afterimages to dissipate. The observers then made posttest judgments of the slide test pattern, followed by judgments of the colors of the computergenerated patterns. The subjects took about 10 min to make the color judgments. There were no restrictions on judgment time; the subjects could bracket the null point by pressing the other button if their judgments seemed to pass the white setting.

Scoring. The scoring of a response as a CAE depended on the prediction from each model. For adaptation stimuli of a red SCB and a green DCB, each model had opposite predictions. For example, the spatial-frequency model predicted that vertical and horizontal gratings would appear red, whereas the edge model predicted a green CAE. For gratings, it was arbitrarily decided that CAEs consistent with the spatial-frequency model would be scored as positive and those consistent with the edge model as negative. With the checkerboard test patterns, a CAE complementary to the adapting stimulus was scored as positive. The opposite color was scored as negative.

\section{Results and Discussion}

The data for the vertical/horizontal and the oblique gratings were analyzed separately using a four-way analysis of variance (ANOVA) with repeated measures. For the vertical/horizontal gratings, the main effect of type of grating (square wave vs. sine wave) was not significant $[F(1,7)$ $=0.04, p>.05$ ], nor were the effects of spatial frequency $(2,2.82,5$, and $7.05 \mathrm{cpd})[F(3,21)=2.02$, $p>.05]$ or orientation of grating (vertical vs. horizontal) $[F(1,7)=0.01, p>.05]$. That is, the CAEs for these gratings were not significant. The mean $\mathrm{CAE}$ scores are set out in Table 1. The results for the oblique gratings were similar. There were no significant main effects of type of grating $[F(1,7)=0.27, p>.05]$, spatial frequency of grating $[F(3,21)=0.99, p>.05]$, or orientation of grating $[F(1,7)=2.92, p>.05]$. In neither analysis were the interaction terms significant. The mean CAE scores are set out in Table 2.

The checkerboard CAE data were analyzed using a three-way ANOVA with repeated measures. There were no significant main effects of checkerboard frequency (2 and $5 \mathrm{cpd})[F(1,7)=0.35, p>.05]$ or type of checkerboard (SCB vs. DCB) $[F(1,7)=0.41, p>.05]$. The interaction of the two also failed to reach significance $[F(1,7)=0.59, p>.05]$. The checkerboard CAE data were analyzed in more detail by means of $t$ tests of difference scores between pretest and posttests. None of the CAE scores was significantly different from zero (Table 3). All the qualitative judgments made on the slide test patterns were white, thus indicating that no CAEs occurred on these patterns.

In summary, no CAEs were present for either test checkerboards or gratings when two checkerboards served as adapting patterns. It should be emphasized that, in Ex- 
Table 1

Mean and Standard Deviations for Vertical and Horizontal Gratings in Experiment 1

\begin{tabular}{|c|c|c|c|c|c|c|c|c|}
\hline \multirow[b]{2}{*}{ Orientation } & \multicolumn{2}{|c|}{2 cpd } & \multicolumn{2}{|c|}{$2.82 \mathrm{cpd}$} & \multicolumn{2}{|c|}{5 cpd } & \multicolumn{2}{|c|}{$7.05 \mathrm{cpd}$} \\
\hline & $M$ & $S D$ & $M$ & $S D$ & $M$ & $S D$ & $M$ & $S D$ \\
\hline \multicolumn{9}{|c|}{ Square-Wave Gratings } \\
\hline Vertical & 1.63 & 4.03 & -0.13 & 1.64 & -0.13 & 1.55 & -2.25 & 2.87 \\
\hline Horizontal & -0.13 & 3.76 & -0.63 & 2.13 & 1.38 & 3.54 & -0.75 & 1.58 \\
\hline \multicolumn{9}{|c|}{ Sine-Wave Gratings } \\
\hline Vertical & 0.25 & 2.55 & 0.00 & 1.93 & 0.25 & 2.05 & -0.63 & 0.74 \\
\hline Horizontal & -1.00 & 1.77 & -0.13 & 1.85 & 0.00 & 2.07 & -0.50 & 1.51 \\
\hline
\end{tabular}

Table 2

Mean and Standard Deviations for Diagonal Gratings in Experiment 1

\begin{tabular}{|c|c|c|c|c|c|c|c|c|}
\hline \multirow[b]{2}{*}{ Orientation } & \multicolumn{2}{|c|}{$2 \mathrm{cpd}$} & \multicolumn{2}{|c|}{$2.82 \mathrm{cpd}$} & \multicolumn{2}{|c|}{$5 \mathrm{cpd}$} & \multicolumn{2}{|c|}{$7.05 \mathrm{cpd}$} \\
\hline & $M$ & $S D$ & $M$ & $S D$ & $M$ & $S D$ & $M$ & $S D$ \\
\hline \multicolumn{9}{|c|}{ Square-Wave Gratings } \\
\hline $\begin{array}{r}45^{\circ} \\
135^{\circ}\end{array}$ & $\begin{array}{r}0.00 \\
-1.25\end{array}$ & $\begin{array}{l}3.67 \\
2.31\end{array}$ & $\begin{array}{l}0.50 \\
0.63\end{array}$ & $\begin{array}{l}1.07 \\
1.51\end{array}$ & $\begin{array}{l}0.38 \\
0.00\end{array}$ & $\begin{array}{l}1.06 \\
1.07\end{array}$ & $\begin{array}{l}2.13 \\
2.25\end{array}$ & $\begin{array}{l}3.60 \\
4.50\end{array}$ \\
\hline \multicolumn{9}{|c|}{ Sine-Wave Gratings } \\
\hline $\begin{array}{r}45^{\circ} \\
135^{\circ}\end{array}$ & $\begin{array}{l}0.25 \\
0.88\end{array}$ & $\begin{array}{l}0.46 \\
2.77\end{array}$ & $\begin{array}{r}0.13 \\
-0.38\end{array}$ & $\begin{array}{l}1.36 \\
1.19\end{array}$ & $\begin{array}{l}-0.75 \\
-0.88\end{array}$ & $\begin{array}{l}1.17 \\
2.10\end{array}$ & $\begin{array}{l}-0.38 \\
-0.13\end{array}$ & $\begin{array}{l}2.50 \\
1.36\end{array}$ \\
\hline
\end{tabular}

periment 1 , we attempted to use some of the stimulus conditions employed by May and Matteson (1976) and Green et al. (1976). We adapted with high-contrast checkerboards projected on a screen by slides, used spatial frequencies that were a close match to the frequencies used in the earlier experiments, and tested with fundamental frequencies $(\sqrt{2} \times$ the checkerboard frequencies) at the appropriate orientations for each checkerboard. Even so, the results did not support either an edge-detector or a spatialfrequency filter model. In addition, we have failed to confirm the spatial-frequency CAE effects achieved with higher spatial frequencies on checkerboards by May, Agamy, and Matteson (1978), but like them, we also failed to produce CAEs with medium frequencies.

In our earlier experiments (Day et al., 1992), we found that the McCollough effect was broadly tuned for spatial frequency. Green (1980) also found that checkerboard aftereffects were broadly tuned for orientation. Kelly (1976) has shown that the two-dimensional spectrum of checkerboards contains fundamental and higher harmonic frequencies located at various orientations from the edges. Given the broadness of the two tuning functions, it is possible that some cancellations of CAEs take place when two checkerboards are adapted, since filters for the various harmonics could overlap in both the spatialfrequency and the orientation dimension. Therefore, in Experiment 2, the role of the higher harmonics in checker- boards and the effects of adaptation with only one checkerboard were investigated.

\section{EXPERIMENT 2}

Kelly (1976) showed that the two-dimensional Fourier transform of a checkerboard pattern is the convolution of a bar-grating spectrum with a similar one rotated by $90^{\circ}$. He showed that there were two pairs of fundamental components in the theoretical spatial-frequency plane of an SCB and a DCB. Each component is oriented at $45^{\circ}$ from the edges of the checks. The frequency of these fundamental components was $\sqrt{2} \times \mathrm{F}$, where $\mathrm{F}$ is the frequency of the two square-wave gratings that are convoluted to produce the spectrum. Kelly (1976) also showed that the other harmonics are widely distributed throughout the spatial-frequency plane. For each checkerboard, there are four pairs of third harmonics at various orientations and with a frequency of $\sqrt{ } 10 \times \mathrm{F}$. There are also four pairs of fifth harmonics for each checkerboard at other orientations and with a frequency of $\sqrt{ } 26 \times \mathrm{F}$.

The aim of Experiment 2 was to produce the McCollough effect by adaptation to both an SCB and a DCB at two frequencies (4 and $0.8 \mathrm{cpd}$ ) and also to adapt each checkerboard by itself. It was predicted from the results of Experiment 1 that selective adaptation would not occur with two checkerboards but a strong CAE would be pro-

Table 3

Means, Standard Deviations, and $\boldsymbol{t}$ Scores for Checkerboards in Experiment 1

\begin{tabular}{lrrrrrrr}
\hline & \multicolumn{3}{c}{2 cpd } & & \multicolumn{3}{c}{5 cpd } \\
\cline { 2 - 4 } \cline { 6 - 8 } Checkerboard & $M$ & $S D$ & $t$ & & $M$ & $S D$ & $t$ \\
\hline Square & -1.75 & 4.55 & -1.09 & & -0.25 & 3.06 & -0.23 \\
Diagonal & 1.00 & 2.56 & 1.10 & & -0.75 & 2.19 & -0.97 \\
\hline
\end{tabular}


duced by adapting only one. A range of test gratings was also used at frequencies and orientations appropriate for fundamental and harmonic components of each checkerboard spectrum. It was anticipated that these stimulus conditions would provide powerful tests of the spatialfrequency and edge-detector models.

\section{Method}

Subjects. Ten undergraduates acted as observers. All had normal color vision, according to the Ishihara (1964) test. Those requiring corrective lenses wore them during the experiment. All were naive regarding the purposes of the experiment.

Apparatus and Procedure. All stimulus patterns were generated on a Barco color monitor (Model CD33), using a real-time image-processing system (Matrox video digitizer, Model PIP-EZ) interfaced to a Super PC (Model AT286). The monitor was mounted in a rotatable cradle, enabling the screen to be set at any orientation to avoid aliasing (Foley \& Van Dam, 1984). The strength of a CAE was measured by color cancellation, using methods already described in detail (Day et al., 1992). Each observer was tested in six sessions, with 1-week intervals between sessions to allow any CAEs induced in an earlier session to dissipate. In Session 1, each observer was adapted for $10 \mathrm{~min}$ with two checkerboards, a green and black SCB ( $4 \mathrm{cpd}, 80 \%$ contrast, $\left.18 \mathrm{~cd} / \mathrm{m}^{2}\right)$ and a red and black DCB ( $4 \mathrm{cpd}, 80 \%$ contrast, $10 \mathrm{~cd} / \mathrm{m}^{2}$ ). Each checkerboard was presented 30 times for $10 \mathrm{sec}$. Six achromatic test stimuli were used during the pretest phase and the posttest phase. Four test stimuli were split field, with a vertical sine-wave grating in the left half field and a horizontal sine-wave grating in the right. Each grating had a different frequency: the frequency of the checkerboards (F), the fundamentals $(\sqrt{ } 2 \times F)$, the third harmonics $(\sqrt{ } 10 \times F)$, and the fifth harmonic $(\sqrt{ } 26 \times F)$. The $F$ and the $\sqrt{2} \times F$ gratings were presented at two pairs of orientations: $0^{\circ}$ and $90^{\circ}$, and $45^{\circ}$ and $135^{\circ}$. The $\sqrt{ } \mathbf{1 0} \times \mathrm{F}$ gratings were presented at four pairs of orientations: $0^{\circ}$ and $90^{\circ}, 18^{\circ}$ and $108^{\circ}, 45^{\circ}$ and $135^{\circ}$, and $27^{\circ}$ and $117^{\circ}$. The $\sqrt{ } 26 \times \mathrm{F}$ gratings were presented at orientations of $0^{\circ}$ and $90^{\circ}, 11^{\circ}$ and $101^{\circ}, 45^{\circ}$ and $135^{\circ}$, and $33^{\circ}$ and $123^{\circ}$. These combinations allowed the frequencies of the Fourier components at their various orientations to be compared with the same frequencies at the orientations of the edges. The last two test stimuli were whole field and consisted of an achromatic SCB and an achromatic DCB. All test stimuli subtended $8^{\circ} \times 7^{\circ}$ and were set at $60 \%$ contrast and $23.4 \mathrm{~cd} / \mathrm{m}^{2}$. A 2-min period was allowed to elapse after adaptation before the posttest was carried out. All pretest and posttest patterns were presented in a random order. In Session 2, each observer was adapted for $10 \mathrm{~min}$ with only an SCB, alternating with a blank screen $\left(0.77 \mathrm{~cd} / \mathrm{m}^{2}\right)$. Stimulus parameters were the same as those in Session 1. The color of the SCB was counterbalanced. The pretest and posttest stimuli were also the same. Gratings at $\sqrt{ } \mathrm{F}$ and $\sqrt{2} \times \mathrm{F}$ were presented at $0^{\circ}$ and $90^{\circ}$ and at $45^{\circ}$ and $135^{\circ}$. Gratings at $\sqrt{ } 10 \times \mathrm{F}$ were presented at $0^{\circ}$ and $90^{\circ}$ and at $18^{\circ}$ and $108^{\circ}$. Gratings at $\sqrt{26} \times \mathrm{F}$ were presented at $0^{\circ}$ and $90^{\circ}$ and at $11^{\circ}$ and $101^{\circ}$. Two achromatic checkerboards were also tested. All procedures were the same as those in Session 1. In Session 3, each observer was adapted for $10 \mathrm{~min}$ with only a DCB counterbalanced for color, alternating with a blank screen. Gratings at $F$ and $\sqrt{2} \times F$ were presented at $0^{\circ}$ and $90^{\circ}$ and at $45^{\circ}$ and $135^{\circ}$. Gratings at $\sqrt{ } 10 \times \mathrm{F}$ were presented at $27^{\circ}$ and $117^{\circ}$ and at $45^{\circ}$ and $135^{\circ}$. Gratings at $\sqrt{26} \times \mathrm{F}$ were presented at $33^{\circ}$ and $123^{\circ}$ and at $45^{\circ}$ and $135^{\circ}$. Two achromatic checkerboards were also tested. All procedures and test stimuli were the same as those for the other sessions.

In Sessions 4, 5, and 6, the stimulus patterns and procedures were the same as those used in Sessions 1, 2, and 3, respectively, except that the frequency of the adapting and test patterns was based on an $F$ of $0.80 \mathrm{cpd}$. For all sessions, an interval of 1 week was found to be long enough for any aftereffect to disappear: no difference was found between pretest judgments after this period.

Scoring. A difference score was calculated by subtracting the pretest score from the posttest score. When the checkerboards were adapted alone, cancellation in the expected color direction was treated as positive. When both SCB and DCB were adapted together,

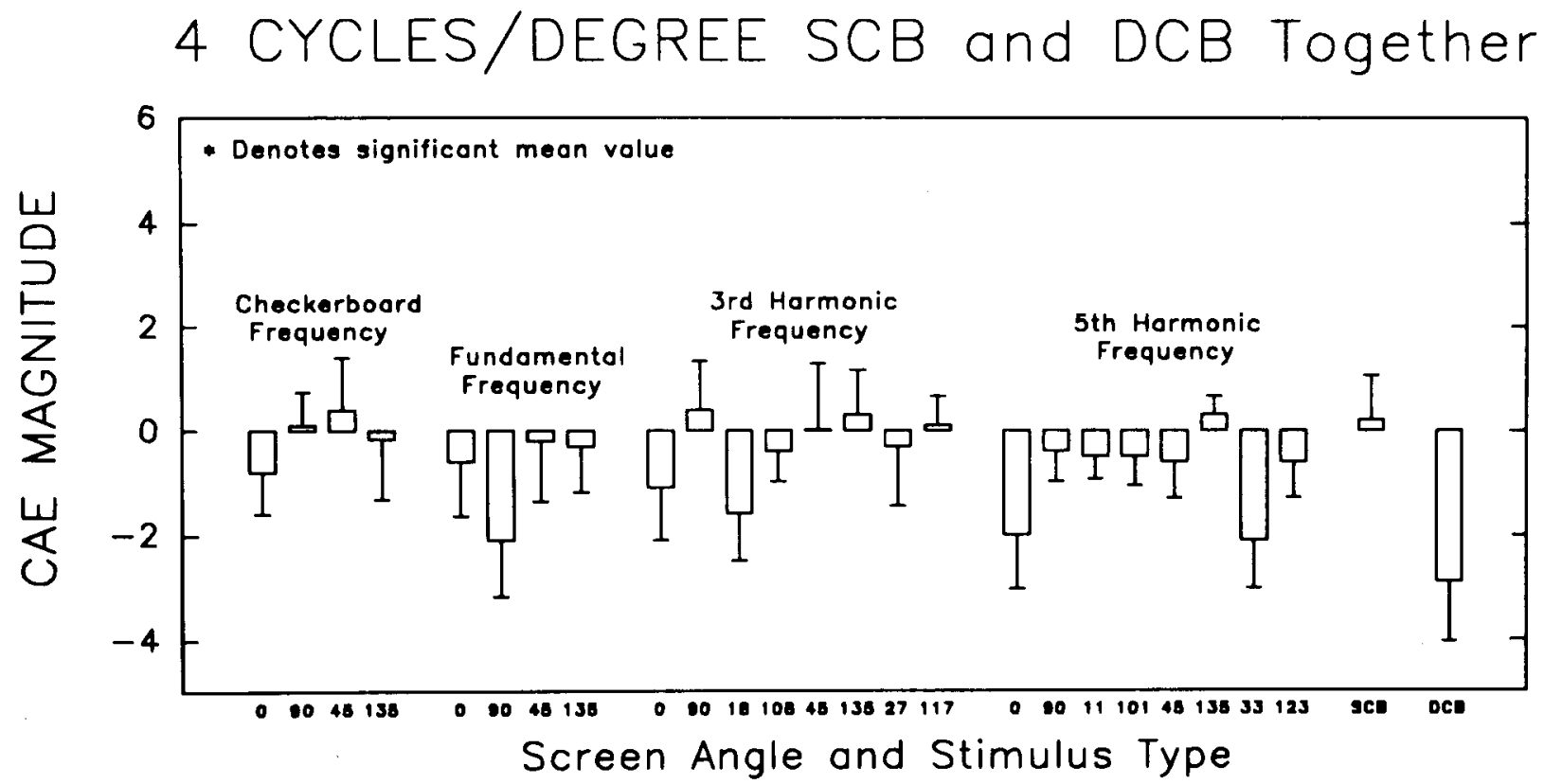

Figure 1. Mean CAE magnitudes after adaptation with square (SCB) and diagonal (DCB) checkerboards at 4 cpd, plotted as a function of screen angle for test gratings and stimulus type (SCB and DCB). The scores for the test gratings are grouped according to the frequency of the components in each checkerboard: checkerboard frequency, fundamental frequency, third harmonic frequency, and fifth harmonic frequency. Error bars represent the standard error of the mean. 
cancellation of green was treated as positive and cancellation of red as negative, since there was no color counterbalancing.

\section{Results}

Session 1. A two-way ANOVA with repeated measures was carried out on the grating data after adaptation of both checkerboards at $4 \mathrm{cpd}$. There were no significant differences between grating means $[F(23,207)=0.87, p>$ $.05]$, and individual $t$ tests revealed no differences between the pretest and posttest scores (see Figure 1). Neither checkerboard mean was significantly different from its pretest (SCB, $M=0.20, t(9)=0.30, p>.05 ; \mathrm{DCB}, M=$ $-2.90, t(9)=2.13, p>.05)$. Thus, adaptation with both checkerboards in alternation failed to produce a selective CAE either on checkerboard or on any of the test patterns.

Session 2. When a 4-cpd SCB was adapted alone, it produced a significant McCollough effect on the test SCB $[M=9.60, t(9)=9.69, p<.01]$, but, surprisingly, it also produced a significant effect of the same color on the test DCB $[M=7.00, t(9)=6.22, p<.01]$ (Figure 2). These two means were also significantly differ- ent $[t(9)=3.40, p<.01]$. A four-way ANOVA with repeated measures was carried out on the test grating data. There was a significant main effect between the gratings at the orientation of the spatial-frequency components and those oriented with the edges $[F(1,9)=7.10, p<.05]$. There was a significant effect due to the frequency of the gratings $[F(3,27)=3.19, p<.05]$. There was also a significant interaction between frequency and orientation predictions for each theory $[F(3,27)=3.60, p<.05]$. There was no significant difference between the left and right test fields $[F(1,4)=0.20, p>.05]$.

Dependent $t$ tests on the difference scores confirmed the results of the ANOVA. Significant CAE effects were found at the orientation of the fundamentals $\left(45^{\circ}\right.$ and $135^{\circ}$ ) for $\mathrm{F}$ gratings and $\sqrt{ } 2 \times \mathrm{F}$ gratings. No significant effects were found at the edge orientations $\left(0^{\circ}\right.$ and $\left.90^{\circ}\right)$ for the same frequencies. Both third harmonics at $18^{\circ}$ and $108^{\circ}$ showed significant effects. The largest effects occurred on gratings at the fundamental frequency, as would be predicted from Fourier analysis (Kelly, 1976). One edge orientation showed a significant effect for $\sqrt{ } 10 \times \mathrm{F}$

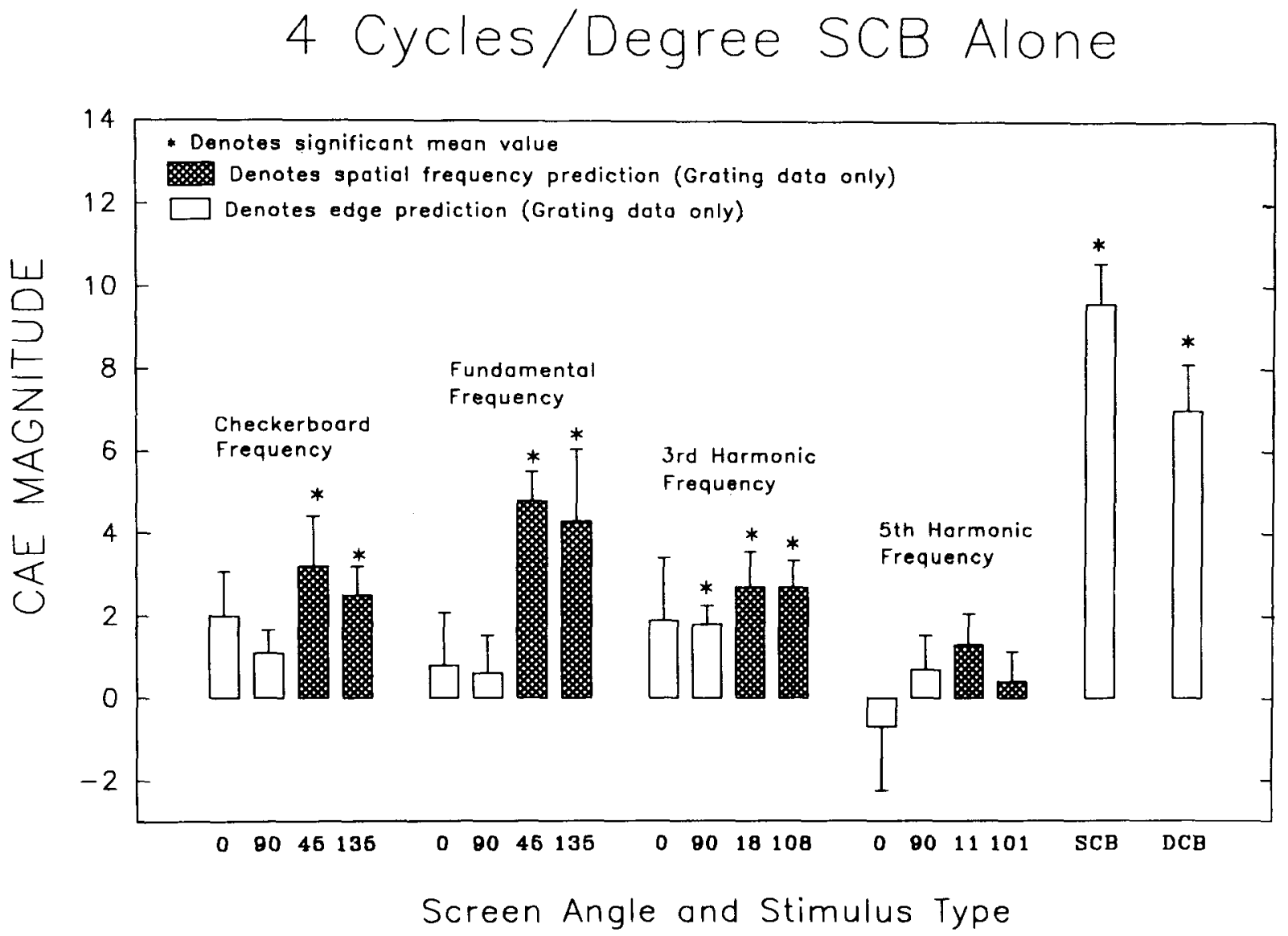

Figure 2. Mean CAE magnitudes after adaptation to a square checkerboard (SCB) of $4 \mathrm{cpd}$, plotted as a function of screen angle for test gratings and stimulus type (SCB and diagonal checkerboard [DCB]). The scores for the test gratings are grouped according to the frequency components of the checkerboard: checkerboard frequency, fundamental frequency, third harmonic frequency, and fifth harmonic frequency. Hatched bars denote screen angles at which CAEs for test gratings would be predicted by spatial-frequency theory. Unfilled bars denote screen angles at which edge-detector theory would predict CAEs. Error bars represent the standard error of the mean. 
at $90^{\circ}$. This effect was small and appeared to be a function of a small standard deviation. Of course, an edge model would not acknowledge the existence of such a frequency in the stimulus array. No effects were observed on gratings at the fifth harmonic frequency $(\sqrt{ } 26 \times F)$.

Overall, these results show that significant McCollough effects occurred on gratings aligned with the Fourier components of the checkerboard spectrum rather than with the edges of the checks.

Session 3. Similar results were obtained with adaptation to the 4-cpd DCB alone (Figure 3). A significant CAE was produced by the test DCB $[M=10.3, t(9)=5.19$, $p<.01]$, and the same CAE was produced by the test SCB $[M=8.9, t(9)=6.08, p<.01]$. These two means do not differ $[t(9)=0.63, p>.05]$. A four-way ANOVA with repeated measures was carried out on the grating data. As with adaptation of the SCB, there was a significant main effect of differences between the gratings at the orientation of spatial-frequency components and those of the edge $[F(1,9)=47.78, p<.01]$. There was also a significant spatial-frequency effect $[F(3,27)=6.11, p<$ $.01]$. There was no significant effect of field location (left or right) or of any of the interactions. Dependent $t$ tests of the difference between pretest and posttest scores confirmed results of the ANOVA. Significant CAEs were observed at the orientations of the spatial-frequency components-that is, on gratings at checkerboard frequency (F) at $0^{\circ}$ and $90^{\circ}$, on gratings of $\sqrt{2} \times \mathrm{F}$ at $0^{\circ}$ and $90^{\circ}$ and on $\sqrt{ } 10 \times \mathrm{F}$ gratings at $117^{\circ}$. One fifth-harmonic mean was significant at $123^{\circ}$. At the fundamental frequency, one mean was significant at the orientation of an edge at $45^{\circ}$. As with adaptation of an SCB, the largest effects occurred at the fundamental frequency that had the greatest power in the checkerboard spectrum (Kelly, 1976). Overall, the results strongly support the view that adaptation of high-frequency checkerboards is mediated largely by Fourier components in the checkerboard spectrum.

Session 4. A two-way ANOVA with repeated measures was carried out on the grating data from the experiment in which both 0.80 -cpd checkerboards were adapted.

\section{CYCLES/DEGREE DCB ALONE}

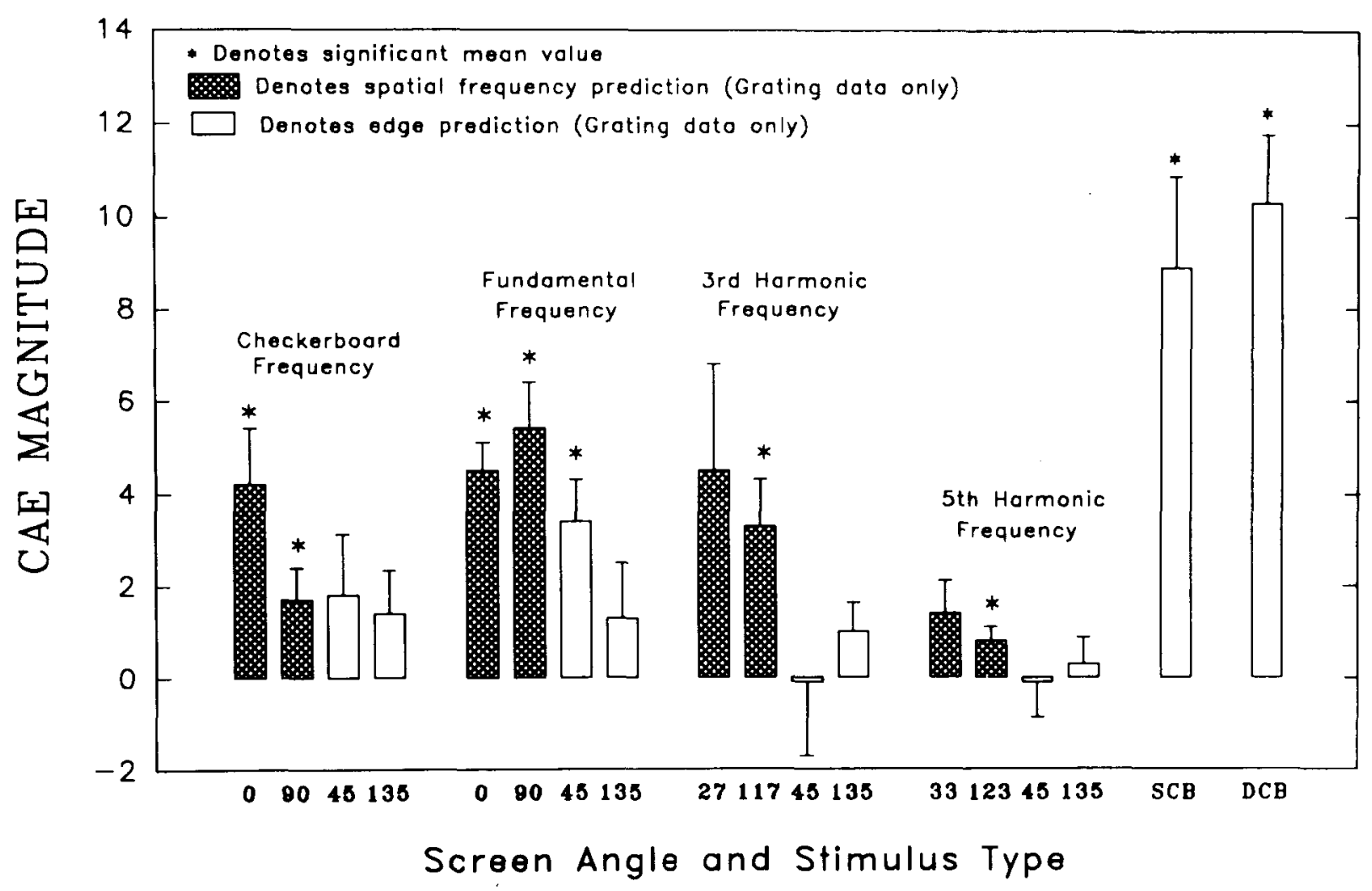

Figure 3. Mean CAE magnitudes after adaptation to a diagonal checkerboard (DCB) of 4 cpd, plotted as a function of screen angle for test gratings and stimulus type (square checkerboard [SCB] and DCB). The scores for the test gratings are grouped according to the frequency components of the checkerboard: checkerboard frequency, fundamental frequency, third harmonic frequency, and fifth harmonic frequency. Hatched bars denote screen angles at which CAEs for test gratings would be predicted by spatial-frequency theory. Unfilled bars denote screen angles at which edge-detector theory would predict CAEs. Error bars represent the standard error of the mean. 
There was no significant difference between test means $[F(23,207)=1.31, p>.05]$ (Figure 4). Dependent $t$ tests showed that none of the test gratings produced a CAE. Neither the test SCB $[M=1.00, t(9)=1.29$, $p>.05]$ nor the test DCB $[M=-0.30, t(9)=0.93$, $p>.05]$ produced a significant CAE. As with adaptation of 4-cpd checkerboards, when both checkerboards were adapted, no selective CAEs were produced.

Session 5. When a 0.80 -cpd SCB was adapted alone, a significant CAE was produced on the test SCB [M= $8.0, t(9)=5.04, p<.05]$ and also on the test DCB $[M=$ $4.1, t(9)=2.69, p<.05]$ (Figure 5). The two means also differed significantly $[t(9)=4.0, p<.01]$. A fourway ANOVA with repeated measures was carried out on the test grating data. Unlike the results with adaptation of a 4-cpd checkerboard, there was no significant effect due to either frequency $[F(3,27)=0.25, p>.05]$ or edge versus spatial-frequency orientation predictions $[F(1,9)=0.00, p>.05]$, but there was a significant interaction between the two $[F(3.27)=5.17, p<.01]$. There was no significant effect due to the left or the right visual field $[F(1,9)=0.11, p>.05]$. Dependent $t$ tests between pretest and posttest scores revealed a mixture of results that supported the ANOVA results (Figure 5). Like adaptation of a 4-cpd SCB, significant spatial-frequency effects were obtained at the checkerboard frequency $(F)$, for the fundamental $(\sqrt{ } 2 \times \mathrm{F})$ at $45^{\circ}$ and $135^{\circ}$ and also for the third harmonic $(\sqrt{ } 10 \times \mathrm{F})$ at $18^{\circ}$ and $108^{\circ}$. However, significant CAEs were also found for the checkerboard frequency $(\mathrm{F})$ at the orientation of the edges $\left(0^{\circ}\right.$ and $90^{\circ}$ ). Thus, it appears that both an edge and a spatialfrequency mechanism are operative under these adaptation conditions. The significant interaction reflects larger effects at some spatial-frequency orientations than at edge orientations.

Session 6. When a 0.80 -cpd DCB was adapted alone, a significant CAE was produced on the test DCB $[M=$ $7.30, t(9)=7.73, p<.011$, and the same CAE was on a test $\operatorname{SCB}[M=5.80, t(9)=6.90, p<.01]$ (Figure 6). These two means also differ significantly $[t(9)=2.42$, $p<.05]$. A four-way ANOVA with repeated measures was carried out on the test grating data. There was no significant effect for frequency $[F(3,27)=2.08, p>.05]$, predicted orientation $[F(1,9)=0.74, p>.05]$, or left

\subsection{Cycles/Degree SCB and DCB Together}

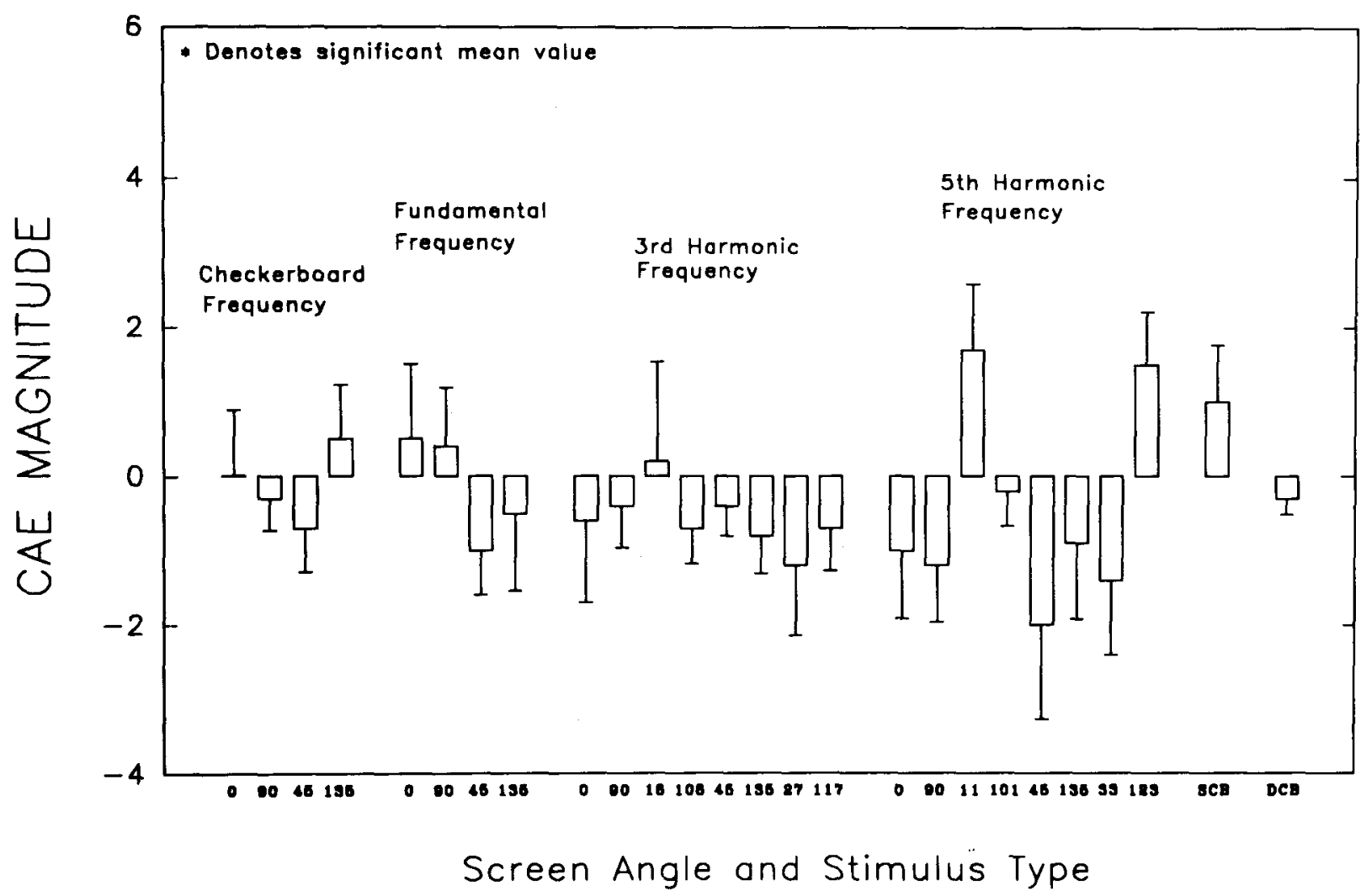

Figure 4. Mean CAE magnitudes after adaptation to both a square checkerboard (SCB) and a diagonal checkerboard (DCB) of 0.8 cpd, plotted as a function of screen angle for test gratings and stimulus type (SCB and DCB). The scores for the test gratings are grouped according to the frequency of the components in each checkerboard: checkerboard frequency, fundamental frequency, third harmonic frequency, and fifth harmonic frequency. Error bars represent the standard error of the mean. 


\subsection{Cycles/Degree SCB Alone}

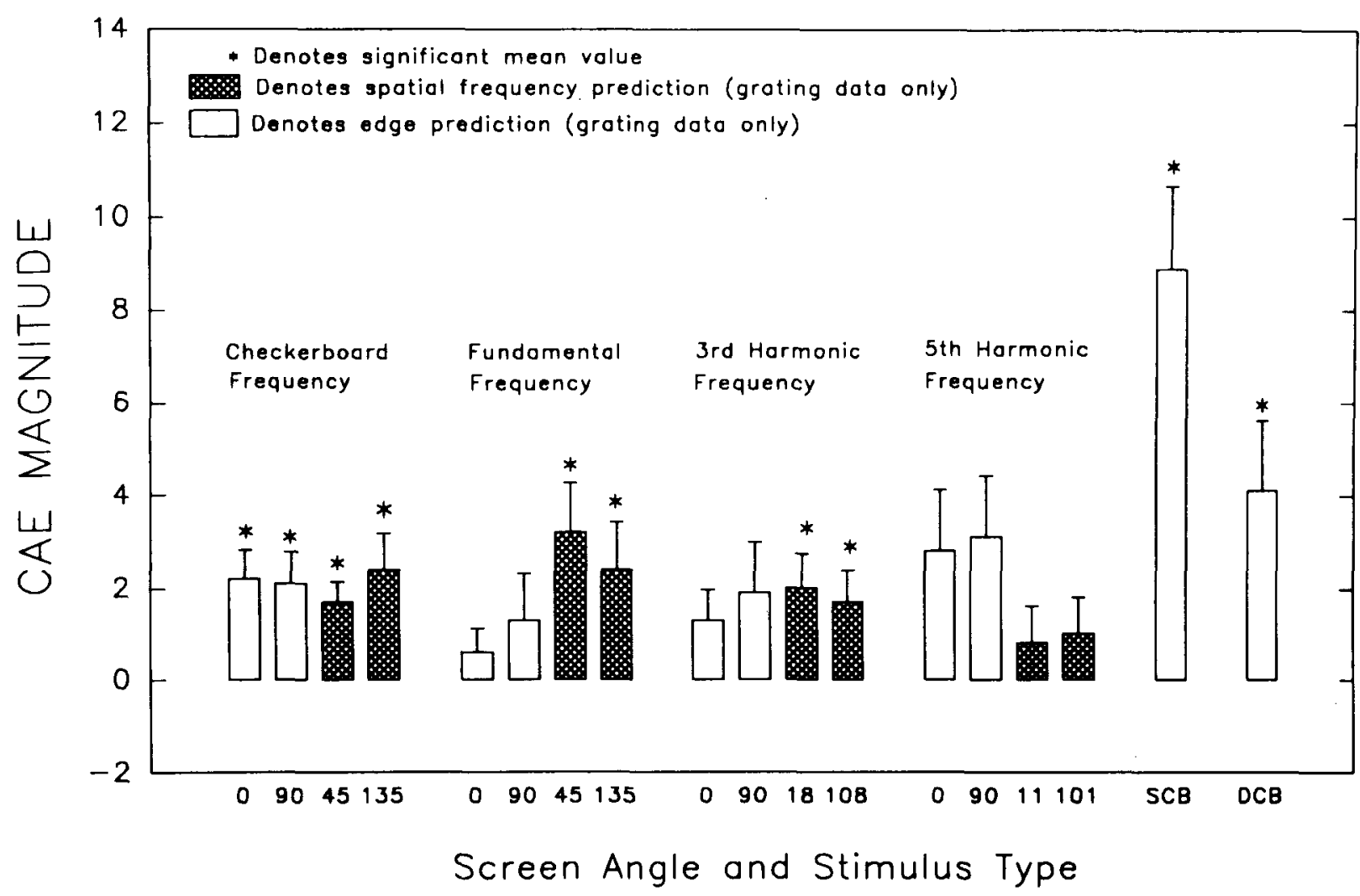

Figure 5. Mean CAE magnitudes after adaptation to a square checkerboard (SCB) of $0.8 \mathrm{cpd}$, plotted as a function of screen angle for test gratings and stimulus type (SCB and diagonal checkerboard [DCB]). The scores for the test gratings are grouped according to the frequency components of the checkerboard: checkerboard frequency, fundamental frequency, third harmonic frequency, and fifth harmonic frequency. Hatched bars denote screen angles at which CAEs for test gratings would be predicted by spatial-frequency theory. Unfilled bars denote screen angles at which edge-detector theory would predict CAEs. Error bars represent the standard error of the mean.

versus right half field $[F(1,9)=1.79, p>.05]$. There were no significant interactions. Dependent $t$ tests carried out on the pretest and posttest scores supported the ANOVA results. CAEs occurred at the checkerboard frequency ( $F$ ) at the orientation of the edges $\left(45^{\circ}\right.$ and $135^{\circ}$ ) and at the orientation predicted by spatial-frequency analysis $\left(0^{\circ}\right.$ and $\left.90^{\circ}\right)$. The only other grating that gave rise to a significant effect was one of the fundamental frequencies $(\sqrt{ } 2 \times \mathrm{F}$ at the orientation of one of the edges [135 $]$ ). These results could suggest that both edge-detector and spatial-frequency mechanisms are operating with checkerboards of $0.80 \mathrm{cpd}$.

\section{Discussion}

The results of Experiment 2 confirm those of Experiment 1: adaptation with two colored checkerboards failed to produce selective-color CAEs on test checkerboards. Between the two experiments, we failed to generate any CAEs on a range of checkerboards $(0.80,2.0,4.0$, $5.0 \mathrm{cpd}$ ) after adaptation with two. In Experiment 1, adap- tation of two checkerboards also failed to produce selectivecolor CAEs, either on test gratings aligned with the edges of the checkerboard or on test-gratings aligned with the fundamentals of the checkerboard spectrum. In this experiment, the observations after adaptation of both checkerboards were extended to show that no selective-color CAEs were produced on gratings aligned with the orientation of the Fourier components of both checkerboards $(\sqrt{ } 2 \times F, \sqrt{ } 10 \times F$, and $\sqrt{2} 6 \times F)$. However, when only one checkerboard was adapted, then, as predicted, strong color CAEs were produced on the adapting checkerboards and on appropriately oriented harmonic gratings. These results indicate that some form of cancellation process could be taking place when both checkerboards are adapted. The results reported by May, Agamy, and Matteson (1978) also suggest a cancellation process in that they failed to generate CAEs on both checkerboards at $0.40,0.80$, and $1.50 \mathrm{cpd}$. They did, however, generate CAEs on checkerboards of $3.0,5.0$, and $7.0 \mathrm{cpd}$. With these higher frequencies, they also found CAEs on square-wave gratings aligned with 


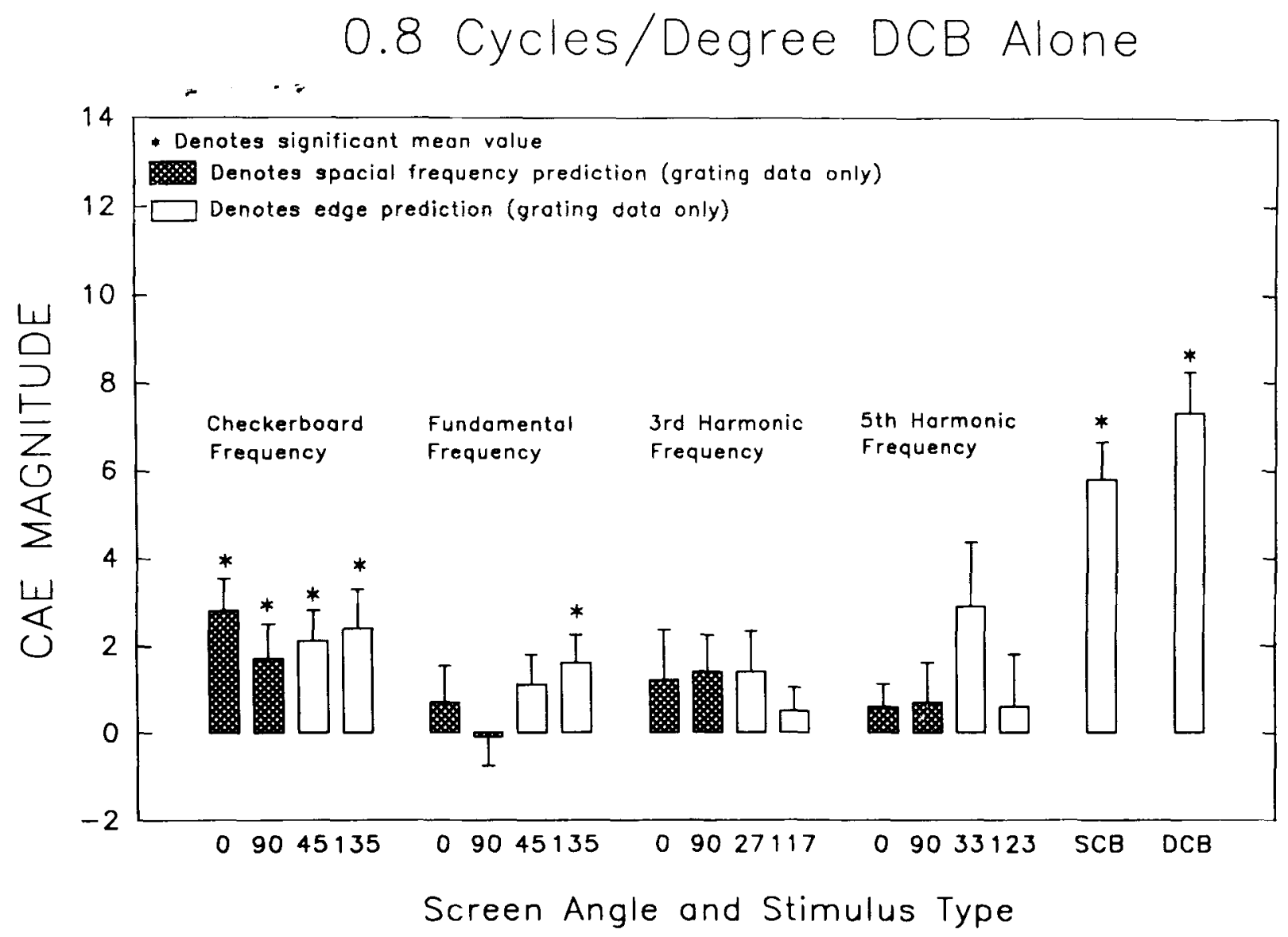

Figure 6. Mean CAE magnitudes after adaptation to a diagonal checkerboard (DCB) of 0.8 cpd, plotted as a function of screen angle for test gratings and stimulus type (square checkerboard [SCB] and DCB). The scores for the test gratings are grouped according to the frequency components of the checkerboard: checkerboard frequency, fundamental frequency, third harmonic frequency, and fifth harmonic frequency. Hatched bars denote screen angles at which CAEs for test gratings would be predicted by spatial-frequency theory. Unfilled bars denote screen angles at which edge-detector theory would predict CAEs. Error bars represent the standard error of the mean.

the fundamentals; however, at $0.80 \mathrm{cpd}$, they found that the CAEs were aligned with the edges. They found no CAEs on gratings of 0.40 and $1.50 \mathrm{cpd}$. Although they interpreted their results to imply that an edge mechanism was operating with 0.80 -cpd gratings, it is odd that they were unable to generate CAEs on any of their other lowfrequency checkerboards. Perhaps, if they had presented sine waves instead of square waves at the harmonic frequencies, they might have obtained results like ours. Overall, our results and some of these reported by May, Agamy, and Matteson (1978) imply that the McCollough effect is based on global activity of linear spatial-frequency filters.

One obvious question is, What role does the oblique effect have in these results? It is well known that visual sensitivity is less for diagonal lines than for vertical or horizontal lines (Campbell, Kulikowski, \& Levinson, 1966). Kelly (1976) tested thresholds of checkerboards for this orientation effect with his contrast-detection method. He found that when vertical gratings and SCBs were rotated through $45^{\circ}$, the thresholds changed in opposite directions. The thresholds of the gratings were increased, whereas those of the checkerboards were decreased. When DCBs were rotated, thresholds changed, but less so. Kelly (1976) concluded that off-axis harmonics partly smooth out the orientation effect. Thus, in our experiments, it is unlikely that the oblique effect could entirely account for the results for DCB adaptation. In the case of SCB adaptation, the significant effects at $45^{\circ}$ from the edges were obtained despite any residual oblique effect.

The most unexpected result in Experiment 2 was that the CAE of the same color could be induced on the adapting checkerboard and on the checkerboard that was not adapted. This occurred on each occasion in which the subjects were adapted to a single checkerboard. On first consideration, this result poses a difficulty for any form of edge-detector model, insofar as the edges of the noninduced grating were never adapted. In fact, these results with the unadapted checkerboard are among the strongest observations in support of a spatial-frequency theory. Only by postulating mechanisms other than edges can a satisfactory account be provided. It would appear that a relatively broad band filtering mechanism resulted in 
CAEs for patterns with edges so far apart. In fact, these observations are not predictable from earlier studies of the McCollough effect with gratings (Stromeyer, 1978). It is usually found that rotation of the head through $45^{\circ}$ will cause the McCollough effect to disappear. Yet an effect was produced on a nonadapted stimulus that had edges located $45^{\circ}$ from the adapting edges. In three out of the four comparisons, the checkerboards that induced adaptation produced a stronger CAE than the one that was not adapted. In the nonsignificant case (Session 3), the difference was in the expected direction, but both CAEs were greater than those produced under the other conditions.

The positive results obtained with harmonics after adaptation to 0.80 - and 4.0-cpd checkerboards lend considerable support to the spatial-frequency theory. Moreover, with 4-cpd checkerboards, gratings presented at the checkerboard frequency $(F)$ produced CAEs only when aligned with the fundamental frequency $(\sqrt{ } 2 \times \mathrm{F})$. After 0.80 -cpd adaptation, gratings at the orientation both of the edges and the fundamentals produced CAEs. This suggests that both an edge and a spatial-frequency mechanism operated. This outcome is unlikely to have been a function of the broad orientation tuning of checkerboards (Green, 1980), because the same separation of orientation $\left(45^{\circ}\right)$ occurred in the 4-cpd checkerboards. Kelly (1976) has suggested that very coarse checkerboards $(0.05 \mathrm{cpd})$ would not show any two-dimensional spatialfrequency effects. He did, however, find effects on coarse checkerboards of $1.40 \mathrm{cpd}$. The present experiments indicate two-dimensional effects down to $0.80 \mathrm{cpd}$, as well as edge effects at this frequency. It would be interesting to examine the McCollough effect with even lower frequency checkerboards. Kelly (1976) also found twodimensional spatial effects with isoluminant checkerboards (red and green) for both fine ( $3 \mathrm{cpd}$ ) and coarse $(1.40 \mathrm{cpd}$ ) checkerboards. These data suggest that two-dimensional McCollough effects might be produced by isoluminant patterns. However, there is some evidence that isoluminant gratings do not generate a CAE (Stromeyer, 1978).

There were some anomalous weak observations at the orientation of the edges. After 4-cpd SCB adaptation, a significant CAE was found at the third harmonic at $90^{\circ}$; after 4-cpd DCB adaptation, a significant fundamental CAE was found at $45^{\circ}$. After 0.80 -cpd DCB adaptation, a significant fundamental $\mathrm{CAE}$ was found at $135^{\circ}$. It is difficult to interpret these results as supporting an edge model, since R. L. De Valois and K. K. De Valois (1980) point out that edge theories do not acknowledge the existence of any such harmonic components apart from the frequency of the edges themselves. Most likely, the broad tuning has spread the effect to these edges to produce weak effects. It is possible that the effects at the third harmonic might also be due simply to the broadly tuned frequency and orientation mechanisms, rather than to a spatialfrequency mechanism. Nevertheless, the significant effects with some fifth harmonics would seem to support a more global explanation.
Other work suggests that there can be reciprocal inhibition between the fundamental and the higher harmonics of square-wave gratings after simple adaptation to squarewave gratings (Nachmias, Sansbury, Vassilev, \& Weber, 1973; Tolhurst, 1972). This was suggested by the finding that adaptation of a square-wave grating elevated the harmonics less than did adapting the harmonics by themselves. The strong harmonic effects achieved here with checkerboards could be due to less reciprocal inhibition being present, because the fundamental and the harmonics now differ in orientation as well as frequency.

\section{GENERAL DISCUSSION}

These results raise problems for Marr's (1982) theory of spatial vision. The first stage in visual processing is described by Marr as the raw primal sketch. The first steps of this sketch are based on a number of bandpass channels or filters (approximately 1.25-octave bandpass). Unlike spatial-frequency processing, the outputs of the different filters are not simply summed in a linear fashion but are processed for zero crossings, by treating them as bandpass second-derivative filters. Marr's proposal is not about the detection of zero values in the output of the filters, but it is about the slope of the zero crossing-that is, the output just to one side of the zero crossing has a peak positive value of the filtered image and just to the other side a peak negative value. Marr's (1982) mechanism for slope detection is that an on-center cell will fire strongly on the positive side and an off-center cell will fire strongly on the negative side. The sum of their firings will correspond to the slope of the zero crossing (in other words, a highcontrast intensity change will generate stronger firing than will a low-contrast change). The theory is basically an edge-detection theory, not a spatial-frequency theory, even though it employs spatial-frequency filters. Marr (1982) argued that "the visual world is not constructed of ripply, wavelike primitives that extend over an area and that add together over it ... by and large the visual world is made of contours"' (p. 69). Marr (1982, p. 76-78) realized that Kelly's (1976) analysis of two-dimensional stimulus patterns, such as checkerboards, presented problems for his theory. He argued that we can clearly perceive the vertical and horizontal edge organization in checkerboards, even though Fourier theory posits no power at these orientations. Using computer modeling, Marr (1982, p. 77) showed that the positive and negative slopes of the zero crossings in checkerboards line up with the edges. Both the positive and the negative slopes have a roughly diagonal orientation in an SCB. Thus, Marr concluded that checkerboards are processed by an edge mechanism, not by summation of linear filters. Logically, this argument has a similar form to those of Smith (1977), Tyler (1977), and MacKay (1981) concerning the diagonal orientation of fundamentals in square checkerboards. As with those arguments, our results with higher harmonics do not support the zero-crossing notion. The orientation of the third 
harmonics would not produce the appropriate aligning of zero-crossing slopes. Hence, our results present real difficulties for Marr's theory of edge detection in checkerboards by zero crossings.

The results reported here have implications for another approach to spatial vision. A global theory of vision has been put forward and tested with the McCollough effect by Emerson, Humphrey, and Dodwell (1988). The basis of this theory is that there are certain "primitive" patterns that underlie most forms of pattern vision and are basic to all visual processing. These higher level structures are a set of vector fields generated by Lie transformation groups (Hoffman, 1966). This theory rejects an explanation of the McCollough effect based on edge detectors because of the long duration of the McCollough effect. Emerson et al. (1988) also reject the spatialfrequency account of the McCollough effect with checkerboards (Green et al., 1976; May \& Matteson, 1976) on the basis of the arguments put forward by Smith (1977) and Tyler (1977) cited above. The results of our experiments with higher harmonics, and particularly the effects obtained on the nonadapted checkerboard, question these arguments and suggest that some form of global spectral mechanism, based on the two-dimensional Fourier analysis of the visual input, takes place. These global processes should be considered before evoking even more global structures, such as Lie vector fields.

If we accept that the results of our experiments indicate that spatial-frequency mechanisms mediate the McCollough effect, the question arises as to where in the visual system the spatial-frequency filters are located. Our work (Day et al., 1992) and earlier work of Stromeyer (1978) and Ellis (1979) suggest that a candidate for the neural mechanism should have the following properties: (1) an opponent-process color system, (2) broad orientation sensitivity, (3) monocular input (since the effect does not show interocular transfer), (4) synaptic connections that can be depressed for long periods of time, (5) broad sensitivity to spatial frequency, and (6) a receptive field that shows linearity of spatial summation to luminance modulations. There is no known class of visual cells that meets all of these criteria. The nearest approximations are simple cells described in primary visual cortex of the primate (K. K. De Valois et al., 1979; R. L. De Valois, Albrecht, \& Thorell, 1982; Michael, 1978). Indeed, Michael (1978) has proposed that simple cells are the mechanisms underlying the McCollough effect. He argues that simple cells "are the only known neurons in the monkey's visual pathway which are monocular, color sensitive and orientation selective. Thus they may be the neural basis of the McCollough effect' (p. 1248). Michael (1978) tested a simple cell with a red-on, green-off central strip flanked by two red-off, green-on surrounds for the McCollough effect. After adaptation with a red-black grating, this cell responded to a white-black grating with off responses (i.e., a green response). Michael showed that the red-black grating selectively adapted the red-sensitive areas in all parts of the receptive field. The cell then became func- tionally one with a green-off central subfield with two green-on surrounds. It was no longer an opponent cell after adaptation and thus now responded to white stimuli.

A considerable problem for the simple-cell model of the McCollough effect is the long duration of the latter (Jones \& Holding, 1975; Riggs, White, \& Eimas, 1974). An alternative explanation of the McCollough effect based on the associative mechanism of classical conditioning has been proposed by Murch (1976). The initiative for developing a conditioning model is the questionable view that contingent aftereffects last much longer than simple aftereffects. But simple visual aftereffects, of the kind used to determine the bandwidths of spatial-frequency filters, can last for hours after protracted adaptation (Blakemore, Nachmias, \& Sutton, 1970; Harris, 1980; Magnussen \& Greenlee, 1985), and other simple aftereffects can last for days (Harris, 1980). These simple aftereffects do not fit readily into a conditioning framework. Furthermore, our finding of the absence of CAEs after adaptation with both checkerboards, does not support a conditioning model. However, it seems that both simple adaptation and CAEs would have to be based on a mechanism that has a long recovery time. There is some relevant neurophysiological evidence about the recovery time of simple cells after achromatic adaptation (Albrecht, Farrar, \& Hamilton, 1984; Maffei, Fiorentini, \& Buti, 1973; Movshon \& Lennie, 1979; Vautin \& Berkley, 1977). Although this neural adaptation was spatial-frequency sensitive, the adaptation periods were short $(30-60 \mathrm{sec})$. The recovery periods matched the duration of the psychophysical recovery periods after similar adaptation periods (Albrecht et al., 1984). A more relevant study was carried out by Creutzfeldt and Heggelund (1975). They adapted adult cats for $1 \mathrm{~h}$ twice a day to achromatic vertical gratings over a period of 2 weeks and found a decrease in the number of visual cortex cells responding to vertical gratings. They did not test formally for recovery, but neurophysiological processes were examined up to $12 \mathrm{~h}$ after the last visual stimulation. These results suggest that a long-term decrease in responding can be produced by long periods of adaptation.

It seems clear that to account for recovery, contingent and simple aftereffects must be postulated on some type of long-term synaptic change. Long-term potentiation has been widely accepted as a neuronal substrate of long-term synaptic change after use (Brown, Chapman, Kairiss, \& Keenan, 1988; Gustafsson \& Wigström, 1988). The evidence for long-term potentiation has come mainly from intracellular recordings in slices taken from either the hippocampus or the cerebellum. Long-term potentiation can last for days and weeks, but there is at present no evidence that it achieves permanence (Gustafsson \& Wigström, 1988). There is now evidence that long-term potentiation is present in both rat (Berry, Teyler, \& Taizhen, 1989) and cat visual cortex (Komatsu, Fujii, Maeda, Sakaguchi, \& Toyama, 1988). Even more recent evidence has shown both long-term potentiation and long-term depression are present in rat visual cortex (Artola, Bröcher, 
\& Singer, 1990). The finding of long-term depression is important in the context of adaptation. This evidence shows that long-term synaptic change can occur in primary visual cortex, as a function of use. Although there is, at present, no evidence as to the type of cell possessing these synaptic changes, it could be possible that simple cells contain these mechanisms.

The McCollough effect has also been discussed in the context of a new theory about how color and spatial stimuli are processed in the primate visual system (Livingstone \& Hubel, 1984). This theory postulates that there are two separate and parallel pathways for color and for spatial stimuli, respectively. Livingstone and Hubel (1984) agree that the McCollough effect indicates that, at some stage in visual processing, orientation and color selectivity converge. But they argue that this convergence does not occur in the "blob" color system, located at VI and VII, because the cells are not orientation sensitive. The "interblob" system does contain some oriented cells (39\%) that are selective for color in VI, but most of these are complex. These data are in striking contrast with other studies of VI (Michael, 1978; Thorell, R. L. De Valois, \& Albrecht, 1984), which have reported many color-coded simple cells that are orientation sensitive. Our results could suggest that simple cells of the interblob system are involved in the McCollough effect.

While the hypothesis about simple cells mediating the McCollough effects is appealing, there are a number of problems. Firstly, is the tuning of CAEs for spatial frequency (criterion 5) and for orientation (criterion 6) too broad for simple cells? Secondly, is it possible that a Fourier system can be based on such broad tuning? A perfect Fourier system would require filters with infinitely narrow bandwidths. The psychophysical bandwidths for simple adaptation ( 0.50 to 1.0 octave) are a reasonable compromise, but R. L. De Valois et al. (1982) found that primate simple cells are tuned to a broad range of spatial frequencies (median $=1.45$ octaves). The distribution of these bandwidths shows a sizable population with bandwidths of 2.0 octaves and larger. They found somewhat similar results for orientation tuning, in that cells broadly tuned for spatial frequency were also broadly tuned for orientation. These values could obviously encompass the tuning for the McCollough effect, but could such broad McCollough detectors mediate a strict Fourier system? Our checkerboard results clearly support the notion that even quite broadly tuned detectors can mediate some sort of global two-dimensional Fourier analysis. Perhaps it is the joint involvement of orientation and spatial frequency that allows this to happen. While our results can be interpreted to support a global Fourier analysis of visual processing, it has been suggested that a much cruder frequency analysis is performed less globally on local patches of the visual field (Kelly \& Burbeck, 1985, p. 156). Conceivably, this process could explain the data reported here. These Gabor patches are based on two pairs of simple-cell receptive fields: symmetrical (cosine transform) and antisymmetrical (sine transform) response profiles (Bishop,
1984; Kulikowski \& Bishop, 1981). In these terms, simple cells are regarded as general-purpose linear cells optimized for processing both spatial-position and spatial-frequency information. To regard them as spatial-frequency analyzers is to stress one side of their function at the expense of the other (Kulikowski \& Bishop, 1981, p. 162). Kulikowski and Bishop suggest that the two processes work in parallel over a limited range of spatial frequencies (1 octave), whereas our results suggest that they might operate over a broader range. The Gabor explanation is a compromise between a narrowband (Fourier) model and a bar-andedge model. Kulikowski \& Kranda (1982) have argued that Gabor-type units have flexible filtering characteristics that allow the extraction of information pertinent to localization, orientation, and the spatial-frequency content of the image. Conceivably, this flexibility could accommodate our two-dimensional results with checkerboards by detecting the presence of harmonics through the joint action of orientation and spatial-frequency mechanisms.

\section{REFERENCES}

Albrecht, D. G., FArrar, S. B., Hamilton, D. B. (1984). Spatial contrast adaptation characteristics of neurones recorded in the cat's visual cortex. Joumal of Physiology, 347, 713-739.

Artolı, A., Bröcher, S., \& Singer, W. (1990). Different voltagedependent thresholds for inducing long-term depression and long-term potentiation in slices of rat visual cortex. Nature, 347, 69-72.

BERRY, R. L., TEYLER, T. J., a TAIZHEN, H. (1989). Induction of LTP in rat primary visual cortex: Tetanus parameter. Brain Research, 481 , 221-227.

Bishop, P. O. (1984). The striate cortex: Feature detection or Fourier analysis. Proceedings of the Australian Physiological \& Pharmacological Society, 15, 1-20.

Blakemore, C., Nachmias, J., \& Sutton, P. (1970). The perceived spatial frequency shift: Evidence for frequency selective neurones in the human brain. Journal of Physiology, 210, 727-750.

Brown, T. H., Chapman, P. F., Kairuss, E. W., Kegnan, C. L. (1988). Long-term synaptic potentiation. Science, 242, 724-728.

Camprell, F. W., Kulikowski, J. J., Levinson, J. (1966). The effect of orientation on the visual resolution of gratings. Journal of Physiology, 187, 427-436.

Creutzfeldt, O. D., Heggelund, P. (1975). Neural plasticity in visual cortex of adult cats after exposure to visual patterns. Science, $188,1025-1027$

Day, R. H., Webster, W. R., Gillies, O., Crassini, B. (1992). Spatial-frequency-contingent color aftereffects: Adaptation with onedimensional stimuli. Perception \& Psychophysics, 51, 57-65.

De Valois, K. K., De Valois, R. L., Yund, E. W. (1979). Responses of striate cortex cells to grating and checkerboard patterns. Journal of Physiology, 291, 483-505.

De Valois, R. L., Albrecht, D. G., Thorell, L. G. (1982). Spatial frequency selectivity of cells in macaque visual cortex. Vision Research, 22, 545-559.

De Valois, R. L., \& De Valois, K. K. (1980). Spatial vision. Annual Review of Psychology, 31, 309-341.

Ellis, S. R. (1979). Orientation selectivity of the McCollough effect: Analysis by equivalent contrast transformation. Perception \& Psychophysics, 22, 539-544.

Emerson, V. F., Humphrey, G. K., Dodwell, P. C. (1988). Coloned aftereffects contingent on patterns generated by Lie transformation groups. Perception \& Psychophysics, 37, 155-162.

FoleY, J. D., \& VAN DAM, A. (1984). Fundamentals of interactive computer graphics. Reading, MA: Addison-Wesley.

GREeN, M. (1980). Orientation-specific adaptation: Effects of checkerboards on the detectability of gratings. Perception, 9, 369-377. 
Green, M., Corwin, T., Zemon, V. (1976). A comparison of Fourier analysis and feature analysis in pattern-specific color aftereffects. Science, 192, 147-148,

Gustafsson, B., Wigström, A. (1988). Physiological mechanisms underlying long-term potentiation. Trends in Neurosciences, 11, 156-162.

HARRIS, C. S. (1980). Insight or out of sight? Two examples of perceptual plasticity in the human adult. In C. S. Harris (Ed.), Visual coding and adaptability (pp. 156-162). Hillsdale, NJ: Erlbaum.

HofFman, W. C. (1966). The Lie algebra of visual perception. Journal of Mathematical Psychology, 3, 65-98.

IsHIHARA, S. (1964). Tests for colour-blindness. Tokyo: Kanehara Shuppan.

JoNEs, P. D., \& Holding, D. H. (1975). Extremely long-term persistence of the McCollough effect. Journal of Experimental Psychology: Human Perception \& Performance, 1, 323-327.

KELLY, D. H. (1976). Pattern detection and the two-dimensional Fourier transform: Flickering checkertoards and chromatic mechanisms. Vision Research, 16, 277-287.

KELLY, D. H. (1979). Manipulation of two-dimensionally periodic stimulus patterns. Behavior Research Methods \& Instrumentation, 11, 26-30.

KeLLY, D. H., \& BurbeCK, C. A. (1985). Critical problems in spatial vision. Critical Reviews of Biomedical Engineering, 10, 125-127.

Kelly, D. H., Magnuski, S. (1975). Pattern detection and the twodimensional Fourier transform: Circular targets. Vision Research, 15, 911-921.

Komatsu, Y., Fuji, K., Maeda, J., Sakaguchi, H., \& Toyama, K. (1988). Long-term potentiation of synaptic transmission in kitten visual cortex. Journal of Neurophysiology, 59, 124-141.

Kulikowski, J. J., BishOP, P. O. (1981). Fourier analysis and spatial representation in the visual cortex. Experientia, 37, 160-162.

KulIKowsk,, J. J., \& Kranda, K. (1982). Image analysis performed by the visual system: Feature versus Fourier analysis and adaptable filtering. In J. J. Pettigrew, K. J. Sanderson, \& W. R. Levick (Eds.), Visual neuroscience (pp. 381-404). Cambridge: Cambridge University Press.

Livingstone, M. S., \& Hubel, D. H. (1984). Anatomy and physiology of a color system in the primate visual cortex. Journal of Neuroscience, 4, 309-356.

MacKay, D. M. (1981). Strife over visual cortical function. Nature, 289, 117-118.

Maffei, L., Fiorentini, A., Buti, S. (1973). Neural correlates of perceptual adaptation to gratings. Science, 182, 1036-1039.

Magnussen, S., \& GreenleE, M. W. (1985). Marathon adaptation to spatial contrast saturation in sight. Vision Research, 28, 1409-1411.

MARR, D. (1982). Vision. San Francisco: W. H. Freeman.
May, J. G., Agamy, G., \& Matteson, H. H. (1978). The range of spatial frequency contingent color aftereffects. Vision Research, 18, 917-921.

May, J. G., Matteson, H. H., Agamy, G., Castellanos, P. (1978). The effects of differential adaptation on spatial frequency-contingent color aftereffects. Perception \& Psychophysics, 23, 409-412.

MAY, J. G., MATTESON, H. H. (1976). Spatial frequency-contingent color aftereffects. Science, 192, 145-147.

McCollough, C. (1965). Color adaptation of edge detectors in the human visual system. Science, 149, 1115-1116.

Michael, C. R. (1978). Color vision mechanisms in monkey striate cortex: Simple cells with dual opponent-color receptive fields. Journal of Neuorophysiology, 41, 1233-1249.

Movshon, J. A., LENNIE, P. (1979). Pattern-selective adaptation in visual cortical neurones. Nature, 278, 850-852.

MURCH, G. M. (1976). Classical conditioning of the McCollough effect: Temporal parameters. American Journal of Psychology, 85, 241-247.

Nachmias, J., Sansbury, R., Vassilev, A., Weber, A. (1973). Adaptation to square-wave gratings: In search of the elusive third harmonic. Vision Research, 13, 1335-1342.

RigGs, L. A., White, D. K., Eimas, P. D. (1974). Establishment and decay of orientation-contingent aftereffects of color. Perception \& Psychophysics, 12, 535-562.

Sмiтh, F. D. (1977). Checkerboards and color aftereffects. Science, 198, 207-208.

Stromeyer, C. F. (1978). Form-color aftereffects in human vision. In R. Held, H. W. Leibowitz, \& H. L. Teuber (Eds.), Perception: Handbook of sensory physiology (Vol. 8). New York: Springer-Verlag.

Thorell, L. G., De Valois, R. L., Albrecht, D. G. (1984). Spatial mapping of monkey VI cells with pure color and luminance differences. Vision Research, 24, 751-769.

ToLHURST, D. J. (1972). Adaptation to square-wave gratings: Inhibition between spatial frequency channels in the human visual system. Journal of Physiology, 226, 231-248.

TYLER, C. W. (1977). Checkerboards and color aftereffects. Science, 198, 208-209.

VAUtin, R. G., \& BerKLey, M. A. (1977). Responses of single cells in cat visual cortex to prolonged stimulus movement: Neural correlates of visual aftereffects. Joumal of Neurophysiology, 40, 1051-1065.

WHITE, K. D. (1976). Luminance as a parameter in the development and testing of McCollough effects. Vision Research, 15, 297-302.

(Manuscript received June 6, 1990; revision accepted for publication August 19, 1991.) 